\title{
Effect of anionic polyelectrolytes on the flow of activated sodium bentonite drilling mud
}

\author{
Kaci Chalah ${ }^{1}$, Abdelbaki Benmounah ${ }^{1}$, Khaled Benyounes ${ }^{2}$ \\ ${ }^{1}$ Unité de Recherche Matériaux-Procédés et Environnement UR-MPE, Université de Boumerdès, Algérie \\ ${ }^{2}$ Laboratoire Génie physique des hydrocarbures, Université de Boumerdès, Algérie
}

\begin{abstract}
Bentonite is often used in water-based drilling fluids. The xanthan gum is widely used as to increase the viscosity of the bentonite suspension. For the stabilization of the drilled layers, we use filtrate reducers: sodium carboxymethylcellulose low viscosity and cellulose polyanionic low viscosity. The objective of this work is to explain the effect of the polymers on the rheological behavior of the $5 \%$ bentonite suspensions. These results will provide practical recommendations for the rational use of different types of additives in water-based drilling muds. Our work is based on rheological trials on a viscometer. The results obtained on the bentonite $5 \%$-xanthane suspension show a rheofluidifying behavior with yield stress conform to the Herschel-bulckly modal. While increasing the concentration of filtrate reducer decreases the yield stress and reduces the viscosity. The effect of CMC LV is more pronounced than PAC L.
\end{abstract}

\section{Introduction}

Dans les fluides de forage à base aqueuse, l'eau constitue le milieu de suspension pour les solides. La bentonite confère au mélange un aspect visqueux lors de son mouvement et thixotropique lorsqu'elle est au repos. On ajoute, éventuellement, des polymères pour améliorer la viscosité, la contrainte seuil et le filtrat. Parmi ces ajout, on cite la gomme xanthane et les dérivés cellulosiques : la cellulose polyanionique faible viscosité (PAC L) et la carboxyméthylcellulose à faible viscosité (CMC LV). La principale mission du fluide de forage est de permettre un forage rapide et sûr pendant le forage [1].

La classification des polymères peut être établie en fonction de la nature des charges portées. De ce fait, le $\mathrm{CMC}$, le PAC et le xanthane sont des polyanions. Selon la structure, ils sont classés respectivement en - Polymère linéaire : pour lesquels chaque chaine macromoléculaire est constituée d'un nombre élevé mais fini d'unités monomères (CMC et $\mathrm{PAC})$ [2]. Un tel système correspond à la polymérisation de monomères bivalents polymère bidimensionnel : ils se présentent sous la forme de feuillets bidimensionnels d'épaisseur comparable à celle des molécules simples (gomme xanthane) [3].

La gomme xanthane est un polyélectrolytique, et comporte un grand nombre de sites ioniques et ayant une continuité des régions d'interactions ioniques. Une fois dissous dans un solvant polaire comme l'eau, le polymère se dissocie, et apparaissent des charges sur son squelette et des contre-ions en solution [4].
Lorsqu'on ajoute un polymère à une suspension d'argile, les interactions mènent à l'adsorption du polymère par les surfaces d'argile. Ce phénomène physique de fixation des chaînes de polymère sur les surfaces de particules d'argile se fait par segments de chaînes appelés "trains". Entre deux trains consécutifs, ces macromolécules forment une "boucle". Le segment de chaîne situé avant le premier train ou après le dernier est appelé "queue" [5].

Une argile en solution aqueuse est reconnue comme étant une suspension fortement anionique $[6,7,8]$.

Par conséquent, le mécanisme d'adsorption d'un polymère cationique sur une surface chargée négativement se fait par interactions coulombiennes entre les groupes cationiques du polymère et la surface d'argile $[9,10,11,12,13]$

Plusieurs auteurs $[14,15,16,17]$ ont abordé l'étude rhéologique et physico-chimique du mélange eau -argile -polymère anionique en prenant en considération l'influence de la salinité. Feret [15] a étudié l'interaction entre le CMC et l'argile en se basant sur l'évolution de l'isotherme d'adsorption, à différentes salinités et concentrations en argile.

\section{Matériels et méthodes}

\subsection{Matériaux et appareillage}

\subsubsection{La bentonite}


La bentonite utilisée provient de gisement de Hamam Boughrara (Maghnia). C'est une bentonite calcique activée au sodium, de couleur blanche, manufacturée par l'entreprise Bental. Une analyse minéralogique par diffraction des rayons $\mathrm{X}$ réalisée sur cette argile a montré une composition cristalline à forte présence de smectite (Na). Nous avons pu identifier également un peu d'illite, des traces de kaolinite, feldspath $(\mathrm{K}$ et $\mathrm{Na})$ et du quartz [18].

\subsubsection{La gomme xanthane}

Le xanthane est un polymère anionique produit à l'échelle industrielle par la fermentation aérobie de la bactérie Xanthomonas campestris. C'est un viscosifiant hydrosoluble, légèrement anionique et à plusieurs branches. Elle est largement utilisée dans le domaine des fluides de forage comme viscosifiant, surtout dans la récupération assistée du pétrole.

Il a un poids moléculaire de 2000 à $3000 \mathrm{Kg} / \mathrm{mol}$. Ces divers groupes fonctionnels (carbonylique, carboxylique, hydroxyle et d'autres) et la longue structure ramifié donnent au xanthane ses propriétés de rhéologie uniques (viscosité apparente et contrainte seuil).

\subsubsection{Les réducteurs de filtrats}

La carboxyméthylecellulose (CMC LV) est utilisée dans les fluides de forage comme un réducteur de filtrat et il peut être aussi utilisée comme un viscosifiant selon sa masse moléculaire. La CMC LV est qualifié de "technique" ou "purifié" suivant la pureté du produit. Dans cette étude, on a utilisé une CMC LV d'une masse moléculaire de $90 \mathrm{~kg} / \mathrm{mol}$ commercialisé par SigmaAldrich, est utilisé.

Le polyanionique de cellulose est disponible en deux catégories (viscosité élevée et faible) qui donnent le même degré de réduction de filtrat mais différent degrés de viscosité. Il est aussi un encapsulant des schistes empêchant le gonflement et la désagrégation. Le PAC L est employé principalement comme réducteur de filtrat pour les boues à base d'eau douce et d'eau de mer.

\subsubsection{Viscosimètre}

Le viscosimètre utilisé est de type couette, le Viscotester VT550, qui contrôle et caractérise des fluides en mode de rotation déterminant leur propriétés rhéologiques à savoir la viscosité, la contrainte seuil. Il consiste à imposer une vitesse de cisaillement et calculer la contrainte de cisaillement et la viscosité apparente. Un régulateur de température de type (HAAKE DC 30) a pour but de maintenir la température voulue. Dans notre cas, tous les essais ont été réalisés à $20^{\circ} \mathrm{C} \pm 1{ }^{\circ} \mathrm{C}$. Ce viscosimètre est menu d'un logiciel RheoWin qui nous permet d'avoir les caractéristiques rhéologiques selon le type de modélisation.

\subsection{Mode préparatoire}

On prépare simultanément une suspension de bentonite-polymère en ajoutant la bentonite à $5 \%$ dans l'eau distillée par petites quantités pour éviter le phénomène d'agrégation des particules. Après $4 \mathrm{~h}$ d'agitation, on ajoute le polymère. Le mélange obtenu est agité pendant 24 heures pour assurer une bonne homogénéisation du mélange et une bonne hydratation du polymère. Un précisaillement de $60 \mathrm{~s}$ sera effectué pour garder le fluide bien homogène, suivi d'un repos de $60 \mathrm{~s}$. La vitesse de cisaillement est de 0 à $700 \mathrm{~s}-1$, effectué pendant une durée de cisaillement de $700 \mathrm{~s}$. De la même façon, on prépare les solutions de bentonite et des polymères en maintenant l'agitation pendant $24 \mathrm{~h}$.

Le fluide à analyser est placé entre deux cylindres de révolution coaxiaux. Pour l'étude des argiles, on a utilisé la géométrie NV. Cette dernière nécessite un faible volume $(9 \mathrm{ml})$, et elle est destinée pour les fluides à faible viscosité.

Les viscosifiants et les réducteurs de filtrats sont ajoutés à différents concentrations $(0.1 ; 0.3$ et 0.5$)$. Dans cette étude, on va mettre en évidence l'action des polyélectrolytes sur le comportement rhéologique du fluide de forage à base d'eau.

\section{Résultats et discussions}

Nous allons précéder en premier lieu à l'étude du comportement rhéologique des solutions des matières premières : la bentonite, le xanthane, le PAC L et la CMC LV. Les résultats obtenus nous servent de base pour mieux approfondir l'effet des polyélectrolytes sur le comportement rhéologique de la solution de bentonite.

Le comportement rhéologique des suspensions de bentonite de Maghnia est décrit par le modèle de Herschel-Bulkley (éq. 1) notamment pour les fortes vitesses de cisaillement. Il devient plus visible à partir de $3 \%$ d'argile [3]. Tandis que les polymères linéaires ont un comportement sans contrainte seuil défini suivant le modèle de Cross (éq. 2).

Le modèle d'Herschel-Bulkley est celui permettant de décrire la plupart des fluides plastiques (fluides à seuil), la courbe d'écoulement de tels fluides finit par devenir rectiligne au-delà d'une certaine contrainte critique appliquée. Il s'agit en fait d'une loi de puissance à laquelle on a ajouté le terme $\tau_{\mathrm{s}}$.

$\tau=\tau_{s}+k \dot{\gamma}^{n}$

où : $\mathrm{K}$ est la consistance, $\gamma$ est la contrainte seuil et $\mathrm{n}$ l'indice d'écoulement.

Le modèle rhéologique de Cross est l'un des plus populaires en service aujourd'hui. Il peut être trouvé pratiquement dans le logiciel d'un rhéomètre, et il peut être utilisé pour extraire les nombres significatifs de l'ensemble de la viscosité et de la vitesse de cisaillement. 


$$
(2)=\eta_{\infty}+\frac{\eta_{0}-\eta_{\infty}}{1+(C \dot{\gamma})^{m}}
$$

où : $\eta_{0}$ est la viscosité au cisaillement zéro, $\eta_{\infty}$ est la Viscosité à l'infini de cisaillement, m est connu sous le nom « constante de taux », C est la constante du temps de Cross.

\subsection{Solutions de bentonite, de PAC L, de CMC LV et de xanthane}

Les résultats d'essais d'écoulements sur les solutions de bentonite, de CMC LV, de PAC L et de xanthane sont illustrés sur la figure $1 \mathrm{a}$ et $1 \mathrm{~b}$. L'analyse de ces courbes montre que :

- Le rhéogramme de la solution de bentonite à $5 \%$ est caractérisé par une contrainte seuil et un comportement rhéofluidifiant. Ce résultat confirme les travaux de Benchabane et Bekkour [19], Luckham et Rossi [20]. En effet le comportement rhéologique des suspensions eaubentonite est décrit par une loi de puissance de type Herschel-Bulkley.

- Nous présentons sur la figure 1a et $1 \mathrm{~b}$ les deux rhéogrammes des solutions PAC L et CMC LV à $0.7 \%$. On y observe que, contrairement aux courbes des suspensions de bentonite et de xanthane, les rhéogrammes se présentent en absence de la contrainte seuil. Conformément à la littérature [21], le comportement rhéologique des solutions de PAC L et CMC LV a été décrit d'une manière satisfaisante par le modèle de Cross.

- la solution de xanthane à $0.3 \%$ montre un comportement rhéfluidifiant avec contrainte seuil. Audessus d'une certaine concentration, les molécules semirigides s'enchevêtrent pour former un réseau stabilisé par des liaisons spécifiques, ponds d'hydrogène, et sont à la base de la structuration responsable du seuil d'écoulement et de la résistance à l'écoulement [22,23]. Pour de fortes vitesses de cisaillement, le réseau est déstructuré et les molécules s'alignent dans le sens du cisaillement en favorisant la diminution de la viscosité $[23,24]$. Le comportement rhéologique est décrit selon le modèle rhéologique Herschel-Bulkley.

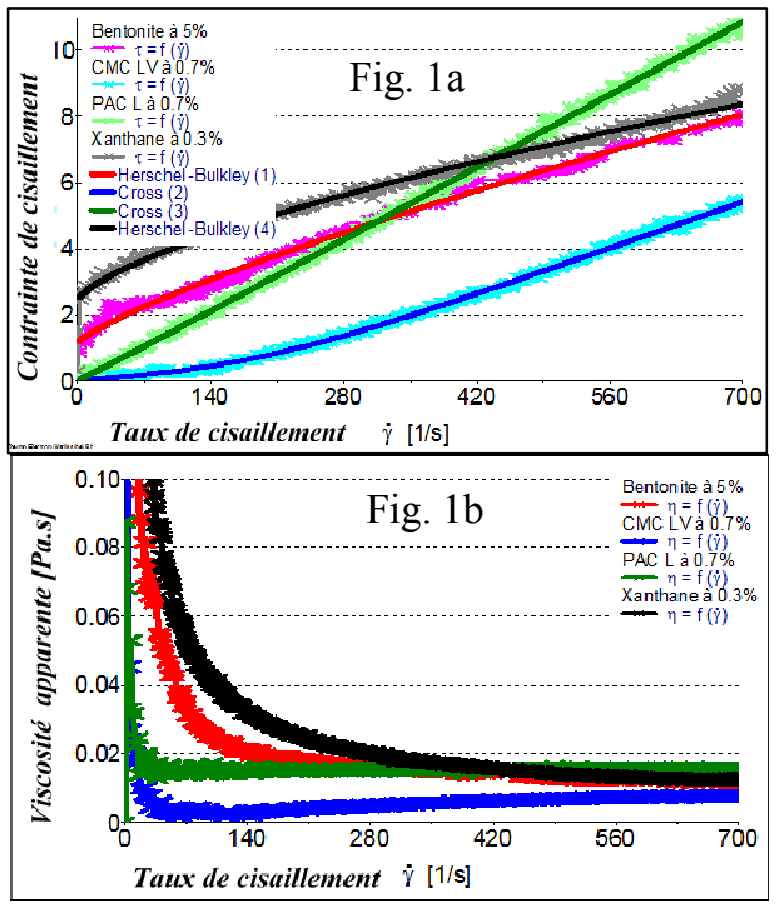

Fig. 1. Comportement rhéologique des Solutions de bentonite de PAC L, de CMC LV et de xanthane : courbes de cisaillement (Fig. 1a) et courbe de viscosité (Fig. 1b)

Les caractéristiques rhéologiques des solutions de bentonite et des polyélectrolytes sont données dans le tableau suivant (Tableau 1). Les courbes de bentonite et de xanthane sont définies par le module d'HerschelBulkley tandis que les rhéogrammes de PAC L et de CMC LV suivent le modèle de Cross.

Tableau 1. Caractéristiques rhéologique des solutions de bentonite de PAC L, de CMC LV et de xanthane

\begin{tabular}{|c|c|c|c|c|}
\hline \multicolumn{5}{|c|}{$\begin{array}{l}\text { Caractéristiques rhéologiques du modèle } \\
\text { d'Herschel-Bulkly }\end{array}$} \\
\hline Essais & $\tau_{0}$ & $\mathbf{K}$ & n & $\mathbf{r}$ \\
\hline BMg à 5\% & 1.127 & 0.0387 & 0.7920 & 0.9954 \\
\hline XAN à $0.3 \%$ & 2.392 & 0.0723 & 0.6742 & 0.9953 \\
\hline \multicolumn{5}{|c|}{$\begin{array}{c}\text { Caractéristiques rhéologiques du modèle } \\
\text { de Cross }\end{array}$} \\
\hline Essais & $\eta_{0}$ & $\eta_{\mathbf{0}}$ & n & $\mathbf{r}$ \\
\hline CMC LV à $0.7 \%$ & 0.0089 & 0.0025 & -2.2210 & 0.9989 \\
\hline PAC L à $0.7 \%$ & 0.0400 & -0.0091 & -0.0231 & 0.9995 \\
\hline
\end{tabular}

\subsection{Mélanges bentonite (5\%)-Polyélectrolytes}

\subsubsection{Mélanges bentonite (5\%)-Xanthane}

La gomme xanthane est caractérisée par sa grande masse moléculaire. Son utilisation augmente la cohérence en rendant le mélange plus résistant au cisaillement. La figure 2 met en évidence le comportement rhéologique du mélange bentonitexanthane.

D'après K. Benyounes et al. [18], les résultats des essais d'écoulements sur les mélanges bentonite (3\%)- 
xanthane, à différentes concentrations de xanthane, montrent que la forme de la courbe des mélanges bentonite-xanthane est semblable à celle des solutions de xanthane avec une augmentation de la viscosité apparente et de la contrainte seuil. Ce résultat est en parfait accord avec une étude réalisée sur un tel système à la base d'une bentonite de laboratoire [23]. On remarque également la disparition du plateau de contrainte observé sur la suspension de bentonite $[23,18]$.

La présence du xanthane à différentes concentrations dans la solution bentonite à $(5 \%)$ a permis d'améliorer les deux paramètres rhéologiques importants à savoir la viscosité et la contrainte seuil. On remarque la disparition de la zone d'agrégation observé sur la suspension de bentonite à $5 \%$, ce qui laisse supposer que le contact entre les particules argileuses n'est plus possible. Dans ce cas, les particules argileuses sont enveloppées par le xanthane et le contact entre les chaînes de polymère va alors former la principale interaction dans le système eaubentonite-xanthane.

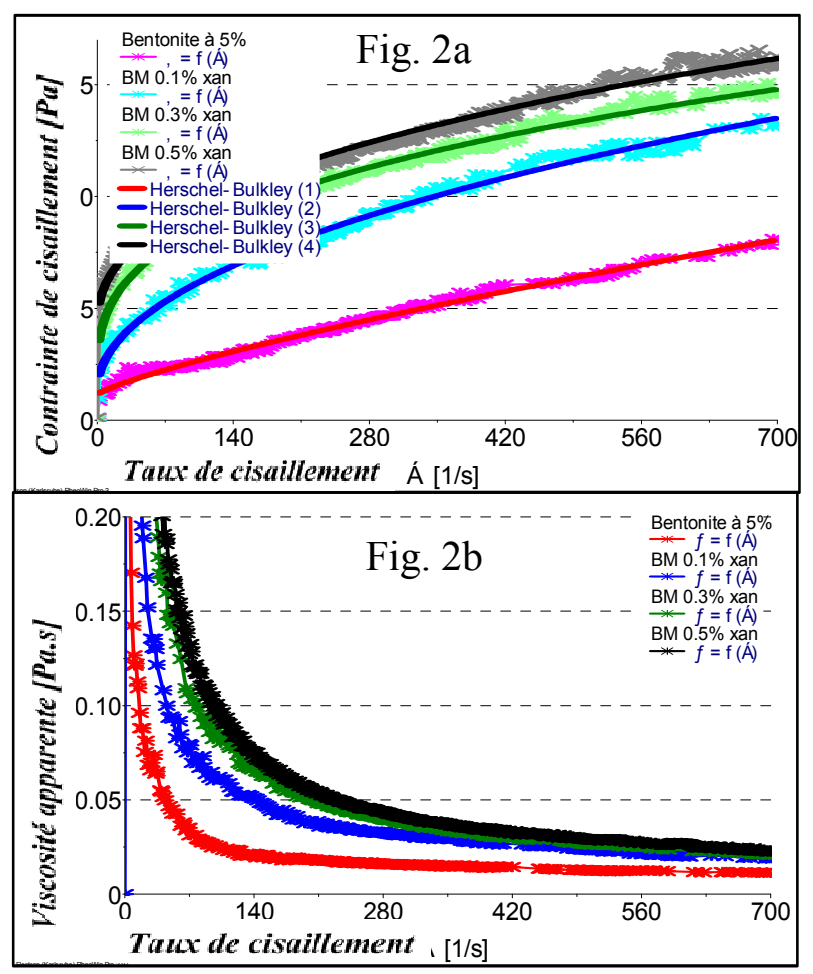

Fig. 2. Comportement rhéologique des Solutions de bentonitexanthane : courbes de cisaillement (Fig. 2a) et courbe de viscosité (Fig. 2b)

L'ajout de $0.1 \%$ de xanthane nous a permet d'améliorer la contrainte seuil de 1,127 à $1.930 \mathrm{~Pa}$ et la consistance de 0.038 à 0.530 (tableau 2), ce qui semble intéressant du point de vue technique et économique.
Tableau 2. Caractéristiques rhéologiques du mélange bentonite (5\%)-xanthane selon le modèle d'Herschel-Bulkley.

\begin{tabular}{|c|c|c|c|c|}
\hline Essais & $\boldsymbol{\tau}_{\mathbf{0}}$ & $\mathbf{K}$ & $\mathbf{n}$ & $\mathbf{r}$ \\
\hline BMg témoin & 1.127 & 0.038 & 0.792 & 0.9954 \\
\hline BMg 0.1XAN & 1.233 & 0.530 & 0.479 & 0.9952 \\
\hline BMg 0.3XAN & 1.644 & 1.445 & 0.337 & 0.9941 \\
\hline BMg 0.5XAN & 4.011 & 0.864 & 0.403 & 0.9902 \\
\hline
\end{tabular}

\subsubsection{Mélanges bentonite (5\%)-PAC L}

Le polyanionique cellulose à faible viscosité (PAC L) est utilisé principalement comme réducteur de filtrat. Son effet sur le comportement rhéologique de la solution de bentonite est montré à la figure 3 .

On peut classer les interactions en deux grands groupes : une interaction de type électrostatique entre les chaînes anioniques de polymère et les charges positives existant sur les bords des particules d'argile et une interaction entre les charges négatives d'argile et le polymère [5].

A la lumière des résultats obtenus, on note une diminution de la contrainte seuil avec l'augmentation de la teneur en PAC L à cause de la neutralisation des charges des particules par les molécules anioniques.

La viscosité diminue à faible quantité en PAC L $(0.3 \%)$ à cause de la neutralisation des forces électrostatiques d'attraction des particules argileuses. A une quantité 0.5 à 0.7 , la viscosité du système augmente par rapport à la viscosité de la suspension eau-bentonite grâce à l'hydratation des particules colloïdales de PAC L.

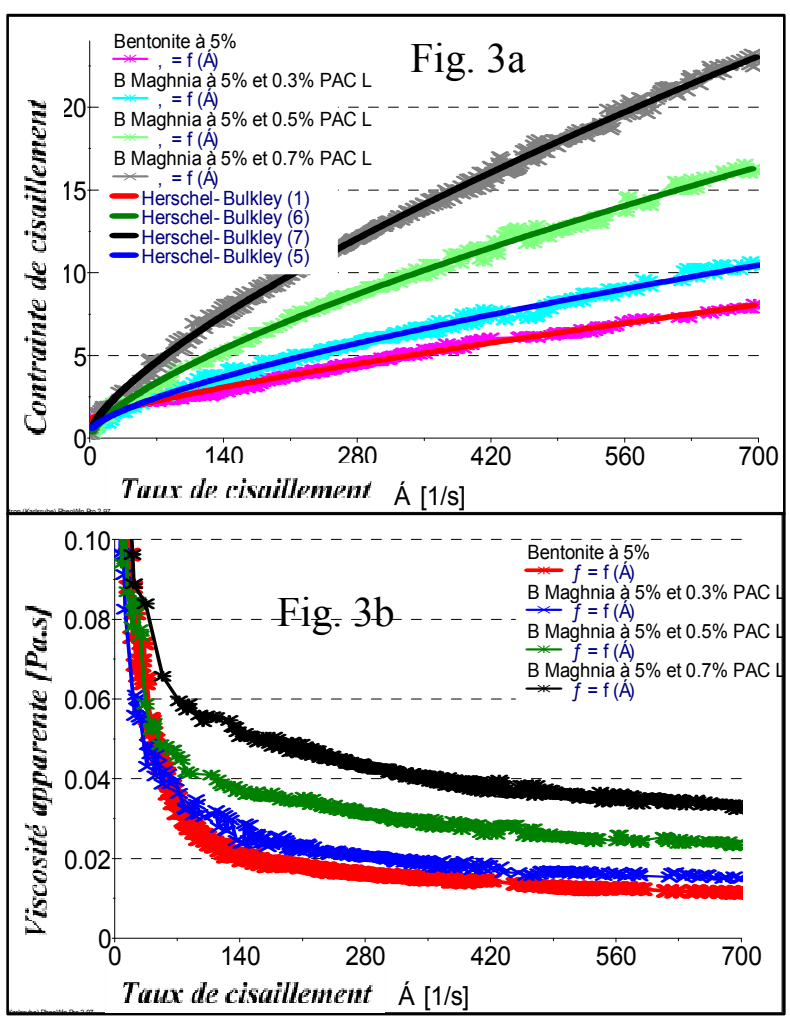

Fig. 3. Comportement rhéologique des Solutions de bentonitePAC L: courbes de cisaillement (Fig. 3a) et courbe de viscosité (Fig. 3b) 
Les caractéristiques rhéologiques du mélange bentonite (5\%)-PAC L sont obtenues selon le modèle d'Herschel-Bulkley. Les valeurs de la contrainte seuil, la consistance du fluide et l'indice d'écoulement sont inscrites dans le tableau suivant :

Tableau 3. Caractéristiques rhéologiques du mélange de bentonite (5\%)-PAC L selon le modèle Herschel-Bulkley.

\begin{tabular}{|c|c|c|c|c|}
\hline Essais & $\boldsymbol{\tau}_{\mathbf{0}}$ & $\mathbf{K}$ & $\mathbf{n}$ & $\mathbf{r}$ \\
\hline BMg témoin & 1.127 & 0.038 & 0.792 & 0.9954 \\
\hline BMg 0.3PAC L & 0.435 & 0.1054 & 0.6949 & 0.9995 \\
\hline BMg 0.5PAC L & 0.091 & 0.1682 & 0.6983 & 0.9977 \\
\hline BMg 0.7PAC L & 0.300 & 0.2017 & 0.7216 & 0.9987 \\
\hline
\end{tabular}

\subsubsection{Mélanges bentonite (5\%)-CMC LV}

La carboxyméthylcellulose à faible viscosité est utilisée principalement comme réducteur de filtrat. Les rhéogrammes obtenue sur le mélange bentonite 5\%-CMC LV montrent la variation de la contrainte seuil et la viscosité.

Le comportement rhéologique de la suspension de bentonite de Maghnia peut être décrit d'une manière satisfaisante par le modèle de Herschel-Bulkley [18]. Selon A. BENCHABANE et K. BEKKOUR [5], les courbes d'écoulement obtenues avec l'ajout de CMC LV sont décrites par le modèle d'Herschel-Bulkley avec une faible contrainte seuil. Les molécules de CMC LV enrobent chaque particule d'argile, empêchant ainsi tout ré-agglomération après cisaillement. Cela explique la diminution de la viscosité.

Les résultats obtenus montrent une diminution de la contrainte seuil et de la viscosité avec l'ajout de CMC LV dans la solution de bentonite (Tableau 4).

L'ajout de la quantité de CMC LV à $0.3 \%$ dans la solution s'accompagne d'une légère augmentation de viscosité, et une forte diminution de la contrainte seuil. Cela s'explique par l'hydratation des particules colloïdales de CMC LV et leur neutralisation des charges des particules argileuses.

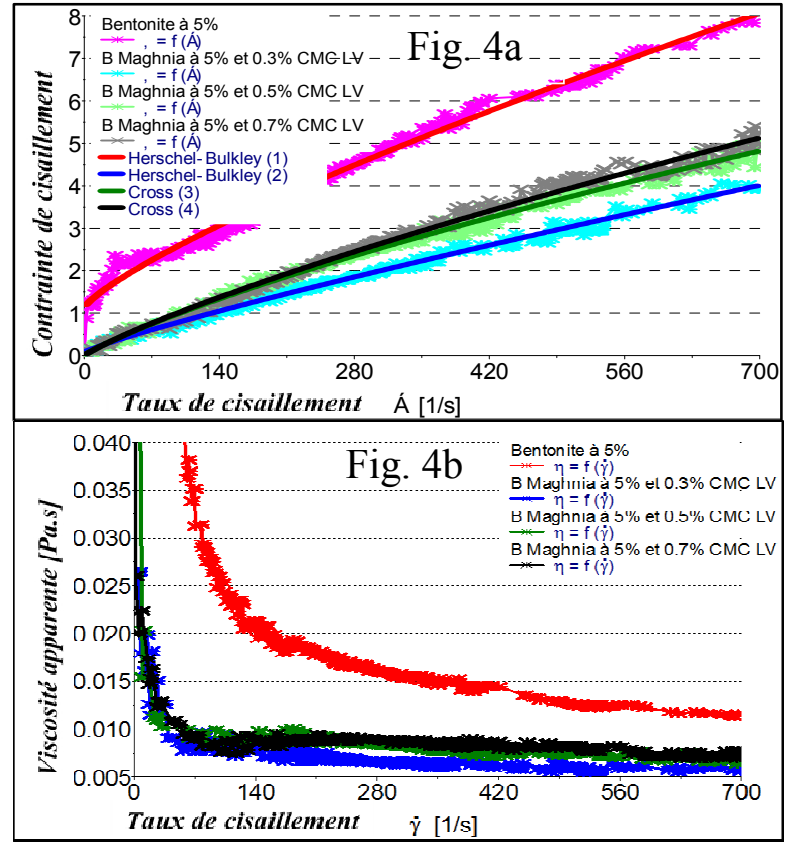

Fig. 4. Impact du CMC LV sur les propriétés rhéologiques de la solution de bentonite à $5 \%$ : rhéogrammes de cisaillement (Fig. 4a) et rhéogrammes de viscosité (Fig. 4b)

Les caractéristiques rhéologiques du mélange bentonite (5\%)-CMC LV sont obtenues selon le modèle d'Herschel-Bulkley et de Cross. La valeur de la contrainte seuil à $0.3 \mathrm{CMC} \mathrm{LV}$ est de $0.061 \mathrm{~Pa}$. A partir de 0.5 à $0.7 \%$, les courbent ne présentent pas une contrainte seuil et sont définies selon le modèle de Cross. La viscosité augmente de 0.3 à $0.7 \%$ mais reste inférieur à la viscosité de la bentonite sans ajout.

Tableau 4. Caractéristiques rhéologiques de la solution de bentonite (5\%)-CMC LV à différentes teneurs en CMC LV

\begin{tabular}{|c|c|c|c|c|}
\hline \multicolumn{5}{|c|}{$\begin{array}{c}\text { Caractéristiques rhéologiques du modèle } \\
\text { d'Herschel-Bulkly }\end{array}$} \\
\hline Essais & $\tau_{0}$ & $\mathbf{K}$ & $\mathbf{n}$ & $\mathbf{r}$ \\
\hline $\mathrm{BMg}$ à $5 \%$ & 1.127 & 0.0387 & 0.7920 & 0.9954 \\
\hline BMg 0.3CMC LV & 0.061 & 0.0149 & 0.8491 & 0.9955 \\
\hline \multicolumn{5}{|c|}{$\begin{array}{c}\text { Caractéristiques rhéologiques du modèle } \\
\text { de Cross }\end{array}$} \\
\hline Essais & $\eta_{0}$ & $\eta_{\oplus}$ & $\mathbf{n}$ & $\mathbf{r}$ \\
\hline BMg 0.5CMC LV & 0.2527 & -0.2342 & 0.0136 & 0.9966 \\
\hline BMg 0.7CMC LV & 0.1273 & -0.1170 & 0.0252 & 0.9951 \\
\hline
\end{tabular}

\section{Conclusion}

L'étude réalisée sur le mélange bentonite (5\%)xanthane montre que l'ajout du xanthane à la solution de bentonite améliore la viscosité et la contrainte seuil. A $0.1 \%$ de xanthane, on note une $\tau_{\mathrm{s}}=1.233 \mathrm{~Pa}$ avec $\eta 100=$ $0.063 \mathrm{~Pa}$.s. Tandis que, pour la solution de bentonite, la $\tau_{\mathrm{s}}$ $=1.127 \mathrm{~Pa}$ avec $\eta 100=0.027 \mathrm{~Pa}$.s. La 2ème zone de la courbe qui correspond à l'agrégation des particules argileuses a totalement disparue. Le modèle d'HerschelBulkley défini bien les courbes obtenues. 
Pour étudier l'influence d'un réducteur de filtrat sur les propriétés rhéologiques de la suspension de bentonite, on a utilisé le CMC LV et le PAC L.

- L'augmentation de la concentration en PAC L de 0.3 à $0.7 \%$ diminue la contrainte seuil de $1.127 \mathrm{~Pa}$ pour la courbe de référence à $0.300 \mathrm{~Pa}$ avec $0.7 \% \mathrm{PAC} \mathrm{L}$. La viscosité se réduit à $0.3 \% \mathrm{PAC} \mathrm{L}$ mais s'améliore avec augmentation à partir de 0.5 à $0.7 \%$.

- L'ajout de CMC LV à la solution de bentonite affecte les propriétés rhéologiques. Les rhéogrammes du mélange de bentonite 5\%-CMC LV, avec des concentrations 0.5 à $0.7 \%$ en $\mathrm{CMC} \mathrm{LV}$, sont définis selon le modèle de Cross. La viscosité augmente de 0.3 à $0.7 \%$ mais reste inférieur à la viscosité de la bentonite sans ajout.

\section{References}

1. H.C. Darley, G.R.Gray, Composition and properties of drilling and completion fluids(Gulf Professional Publishing, Texas, 1988)

2. O.H. Houwen, SPE Drilling \& Completion 8, (1993)

3. M. Fontnille, Y. Gnanou, chimie et physicochimie des polymères( Dudon, Paris, 2002)

4. W.Xie, J.Lecourtier, Polym. Degrad. Stab. 38, (1992)

5. A. Benchabane, K. Bekkour, 39ème Colloque annuel du GFR, pp. 201-205 (2004)

6. Y.Deng, J.B.Dixon, G.N.White, R.H. Loeppert, A.S.R. Juo, Colloids Surf., A 281, ( 2006)

7. A. McFarlane, K.Y. Yeap, K. Bremmell, Colloids Surf., A 317, (2008)

8. P. Mpofu, J. Addai-Mensah, J. Ralston, Int. J. Miner. Process. 71, (2003)

9. A. Alemdar, N. Öztekin, N. Güngör, ÖI. Ece Colloids Surf., A 252, (2005)

10. C. Breen, Appl. Clay Sci. 15, (1999)

11. J. Dau, G. Lagaly, Croatica chemica acta 71, (1998)

12. S. İşçi, F.S. Güner, ÖI. Ece, N. Güngör, Prog. Org Coat. 54, (2005)

13. M. Janek, G. Lagaly, Colloid. Polym. Sci. 281, (2003)

14. A. Alemdar, N. Güngör, F.B. Erim, J. Mater .Sci. Lett. 22,(2003)

15. B. Feret,doctorat de l'Université Pierre et Marie Curie, France,(1992).

16. N. Güngör, A. Alemdar, J. Incl. Phenom. Macro. 33, (1999)

17. O. M'bodj, N.K. Ariguib, M.T. Ayadi, A. Magnin, J Colloid Interf Sci 273,(2004)

18. K.Benyounes, A. Benchabane, A. Mellak, Energ Source Part A 32, (2010)

19. S.Kouloughli, M. B. Lehocine, Sci.Techn.B 25, (2007)

20. P.F. Luckham, S. Rossi, Adv Colloid Interfac 82, (1999)

21. A. Benchabane, K. Bekkour, Colloid Polym. Sci. 286, (2008)
22. A. Benchabane, Thèse de doctorat de l'ULP Strasbourg France, (2006).

23. A. Benchabane, K. Bekkour ,Rheol. Acta 45, (2006)

24. K.W. Song, Y.S. Kim, G.S. Chang, Fiber Polym 7, (2006) 